\title{
A mini updated review on the role of coffee in non- alcoholic fatty liver disease
}

\author{
Abstract \\ Non-alcoholic fatty liver disease (NAFLD) is dramatically increasing worldwide with \\ detrimental effects to the health. Coffee has been shown to have a beneficial effect on \\ NAFLD. This mini review article will bring you to most updated information on the \\ role of coffee in NAFLD in general population. \\ Keywords: caffeine, non-alcoholic fatty liver disease, effects; adults, insulin \\ resistance, prospective evaluations, evidence on coffee and nafld, effect on fibrosis, \\ insulin sensitivity, anxiety, restlessness, caffeine, theobromine, paraxanthine, \\ theophylline
}

Volume 3 Issue 3 - 2017

\author{
Dimitrios Papandreou \\ Zayed University, UAE
}

Correspondence: Dimitrios Papandreou, Department of Natural Science and Public Health, Zayed University, Khalifa B City,Abu Dhabi 144534, United Arab Emirates, Tel 97125993677, Email papandreoudimitrios@yahoo.gr

Received: April 07, 2017| Published: May 03, 2017
Abbreviations: NAFLD, non-alcoholic fatty liver disease; $\mathrm{NASH}$, non-alcoholic steatohepatitis; CGA, chlorogenic acid

\section{Introduction}

Non-Alcoholic Fatty Liver Disease (NAFLD) is termed as the accumulation of extra fat in hepatic cells and it is mainly associated with obesity and insulin resistance. ${ }^{1}$ NAFLD is very common in the general population and almost up to $29 \%$ of the adults are documented with excessive fat accumulation in the liver. ${ }^{2}$ NAFLD encompasses a histological spectrum ranging from simple steatosis to non-alcoholic steatohepatitis (NASH). Currently, the pooled mean prevalence of NAFLD in adults from general population studies is $25 \%$ (95\%CI: $22 \%$ to $29 \%$ ) with highest in the Middle East and South Africa and lowest in Africa. ${ }^{3}$

Coffee is the most commonly consumed beverage in the world and there has been a lot of interest in its beneficial effects on health. Coffee is a very rich source of antioxidants and the protective effects of coffee have been proposed in a variety of conditions ranging from heart disease to stroke to type 2 diabetes, as well as Parkinson disease. ${ }^{4}$ Coffee and tea contain a wide variety of other chemicals with potentially bioactive properties such as caffeine, including other alkaloids (theobromine, paraxanthine, and theophylline) and polyphenols (tannins and flavonoids (chlorogenic acid). ${ }^{5}$ Caffeine is an alkaloid xanthine derivative (1,3,7-trimethylxanthine) found in, and added to, a wide variety of foods and beverages. Most of the caffeinated beverages consumed throughout the world contain caffeine extracted from coffee beans or tea leafs, but caffeine is also eaten as chocolate derived from the cacao bean. ${ }^{5}$

\section{Mechanism of action and dose safety}

The exact mechanism of the beneficial effects of coffee is not clear yet. Coffee contains more than 1000 substances with the most important one, the chlorogenic acid (CGA) that has been found to decrease NAFLD development in rats by modulating glucose intolerance. ${ }^{6}$ In addition, coffee has been found to reduce fibrosis in animal tissue by decreasing the expression of growth factor-b, which is responsible for the connective tissue growth factor that contributes to increase accumulation of fat in hepatic cells. ${ }^{7}$ Furthermore, many studies have suggested the caffeine consumption plays a protective role in NAFLD. ${ }^{8-10}$
The beneficial effects of coffee are reported for 2 cups/day. One cup is equivalent to $10 \mathrm{~g}$ of whole bean coffee and $5 \mathrm{~g}$ of instant coffee. Incremental beneficial effects have been reported up to 4-6 coffee cups a day. However, coffee drinking in pediatric age group should be discouraged in view of side effects of caffeine in form of anxiety, restlessness, etc. Up to $400 \mathrm{mg}$ of caffeine a day is considered safe. ${ }^{11}$

\section{Coffee and NAFLD/NASH}

The available experimental, as well as clinical, evidence suggests that coffee consumption has protective effects against metabolic syndrome, as well as development of NAFLD. The clinical evidence for beneficial effect of coffee against NAFLD is also overwhelming. There are published population-based case- control studies and population based cohort, as well as cross-sectional studies, supporting the usefulness of coffee in NAFLD. In a most recent meta-analysis by Marventano et al. ${ }^{12}$ the authors concluded that coffee consumption has a protective role on fibrosis. ${ }^{12}$ The aforemented authors analyzed 7 studies out of 252 that included specific criteria. The first study was from Catalano et al. ${ }^{10}$ that evaluated 157 patients with NAFLD and 153 controls. Although no difference in coffee intake was observed between cases and controls, among NAFLD patients the number of cups of coffee/day was inversely associated with bright liver score. ${ }^{10}$ The second study came from the National Health and Nutrition Examination Surveys that enrolled 18,550 subjects. The authors concluded that 1782 individuals with NAFLD indicated caffeine intake as an inverse and independent predictor of NAFLD, suggesting a potential protective effect. ${ }^{13}$ Molloy et al. ${ }^{14}$ aimed to evaluate the association of coffee and caffeine consumption with prevalence and severity of biopsy proven NAFLD. The authors suggested a more important role of whole coffee, rather than total caffeine consumption, in preventing the progression of fibrosis in NASH. In another case control study from Mexico 130 subjects participated in a study investigating the potential antioxidant effect of coffee showed a dose-dependent reduction in caffeine intake with increasing severity of steatosis (diagnosed by ultrasonography), although no significant difference in antioxidant enzymes and lipid peroxidation markers was found. ${ }^{15}$ Furthermore, a French study in 195 bariatric patients with biopsy-proven NAFLD showed that the consumption of regular coffee was an independent protective factor for liver fibrosis. ${ }^{9}$ In addition, 782 adults from the Nonalcoholic Steatohepatitis Clinical Research. 
Network participated in investigating the role of coffee in NAFLD and the results showed that coffee was inversely associated with fibrosis in subjects with a low insulin resistance, while no protective effect was found in subjects with higher HOMA-IR. The authors suggested that the protective effects of coffee may be due to its effects on improving insulin sensitivity, and this effect could be overshadowed in subjects with higher insulin resistance. ${ }^{8}$ Finally, a recent Israeli study evaluated the effects of coffee intake on NAFLD in a cohort of 347 and subgroup of 147 patients who were randomly sampled from the national population registry and interviewed in the First Israeli National Health and Nutrition Survey (the MABAT Survey). The authors found that coffee consumption was not associated with NAFLD prevalence and incidence in both cross-sectional and prospective evaluations, respectively. On the other hand, high coffee consumption ( $>3$ cups/d) was significantly associated with lower risk of clinically significant fibrosis. ${ }^{16}$

\section{Summary}

Coffee is beneficial for health in general and particularly for patients with liver disease. The incidence of advanced fibrosis and cirrhosis is lower among coffee drinkers. Most of the evidence on coffee and NAFLD suggest that coffee has a protective effect on fibrosis. However, more interventional studies are needed to confirm this relationship.

\section{Acknowledgements}

None.

\section{Conflict of interest}

Author declares that there is no conflict of interest.

\section{References}

1. Loria P, Lonardo A, Carruli L, et al. Review article: the metabolic syndrome and non-alcoholic fatty liver disease. Aliment Pharmacol Ther. 2005;22(Supp 2):31-36.

2. Browning JD, Szczepaniak LS, Dobbins R, et al. Prevalence of hepatic steatosis in an urban population in the United States: impact of ethnicity. Hepatology. 2004;40(6):1387-1395.

3. Younossi ZM, Koening AB, Abdelatif D, et al. Global epidemiology of nonalcoholic fatty liver disease-Meta-analytic assessment of prevalence, incidence, and outcomes. Hepatology. 2016;64(1):73-84.
4. Adriana Farah. Coffee constituents. In: Chu Y-F, editor. Coffee: Emerging Health Effects and Disease Prevention. 1st edn. USA: Blackwell Publishing Ltd; 2012:21-58.

5. Gomez Ruiz JA, Leake DS, Ames JM. In vitro antioxidant activity of coffee compounds and their metabolites. J Agric Food Chem. 2007;55(17):6962-6969.

6. Panchal SK, Poudyal H, Waanders J, et al. Coffee extract attenuates changes in cardiovascular function and hepatic structure and function without decreasing obesity in high-carbohydrate, high-fat diet-fed male rats. J Nutr. 2012;142(4):690-697.

7. Arauz J, Moreno MG, Cortes Reynosa P, et al. Coffee attenuates fibrosis by decreasing the expression of TGF-b and CTGF in a murine model of liver damage. J Appl Toxicol. 2013;33(9):970-979.

8. Bambha K, Wilson L, Unalp A, et al. Coffee consumption in NAFLD patients with lower insulin resistance is associated with lower risk of severe fibrosis. Liver Int. 2014;34(8):1250-1258.

9. Anty R, Marjoux S, Iannelli A, et al. Regular coffee but not espresso drinking is protective against fibrosis in a cohort mainly composed of morbidly obese European women with NAFLD undergoing bariatric surgery. J Hepatol. 2012;57(5):1090-1096.

10. Catalano D, Martines G, Tonzuso A, et al. Protective role of coffee in non-alcoholic fatty liver disease (NAFLD). Dig Dis Sci. 2010;55(11):3200-3206.

11. Winnie Wing Man Leung, Suzanne C Ho, Henry LY Chan, et al. Moderate coffee consumption reduces the risk of hepatocellular carcinoma in hepatitis B chronic carriers: a case-control study. Journal of Epidemiology and Community Health. 2011;65(6):556-558.

12. Marventano S, Salomone F, Godos J, et al. Coffee and tea consumption in relation with non-alcoholic fatty liver and metabolic syndrome: A systematic review and meta-analysis of observational studies. Clin Nutr. 2016;35(6):1269-1281.

13. Birerdinc A, Stepanova M, Pawloski L, et al. Caffeine is protective in patients with non-alcoholic fatty liver disease. Aliment Pharmacol Ther. 2012;35(1):76-82.

14. Molloy JW, Calcagno CJ, Williams CD, et al. Association of coffee and caffeine consumption with fatty liver disease, nonalcoholic steatohepatitis, and degree of hepatic fibrosis. Hepatology. 2012;55(2):429-436.

15. Gutierrez Grobe Y, Chavez Tapia N, Sanchez Valle V, et al. High coffee intake is associated withlower grade nonalcoholic fatty liver disease: the role of peripheral antioxidant activity. Ann Hepatol. 2012;11(3):350-355.

16. Zelber Sagi S, Salomone F, Webb M, et al. Coffee consumption and nonalcoholic fatty liver onset: a prospective study in the general population. Transl Res. 2015;165(3):428-436. 